\title{
Christoph Görg
}

\section{Plädoyer für Gesellschaftstheorie. Eime Replik auf Christoph Scherrer}

Manche Debatten entfalten eine erstaunliche Eigendynamik. Angetrieben von Fragestellungen, die weit über ihren direkten Gegenstand hinausreichen und über die auf dem ursprünglich strittigen Terrain kein Konsens zu erzielen ist, neigen sie dazu, sich zu verselbständigen. Sie entfalten ein geisterhaftes Eigenleben, führen bei einsetzender Gewöhnung aber auch zu Ermüdungserscheinungen. Ohne zu einer wirklichen Klärung gefühtt zu haben schlafen sie ein oder werden auf einem anderem Terrain fortgeführt. Von diesen Merkmalen scheinen auch die Debatten um die handlungs- und staatstheoretischen Defizite der Regulationstheorie geprägt zu sein. Seit Mitte der 80er Jahre in verschiedenen Varianten immer wieder diagnostiziert dürfte dieses Defizit, wie Christoph Scherrer zu Recht bemerkt hat, inzwischen fast genauso bekannt sein wie die Arbeiten aus der Regulationstheorie selbst. Im folgenden sollen daher weder ein weiteres Mal diese Defizite demonstriert noch eigentlich versucht werden, sie zu überwinden. Vielmehr hat sich inzwischen gezeigt, daß eine Lösung auf dem ursprünglichen Terrain nicht möglich, eine Verbindung mit Lösungsansätzen aus anderen theoretischen Traditionen die strittigen Punkte, die auch schon auf dem Boden der Regulationstheorie viel mit unterschiedlichen Theorieansätzen zu tun haben, dagegen noch vervielfachen.

Zu fragen wäre vielmehr, was denn eine »Lösung « der mit dem Problem des Verhältnisses von Handlung und Struktur eher chiffrenhaft benannten Defizite überhaupt bedeuten würde. Zum einen beinhaltet dieses Verhältnis nämlich einen ganzen Komplex sehr unterschiedlicher begrifflicher, konzeptueller und geschichtlicher Fragestellungen, die zudem je nach theoretischem Ausgangspunkt völlig unterschiedlich interpretiert und zum Teil überhaupt nicht als Problem wahrgenommen werden. Sollte unter einer »Lösung « ein Konsens über diese Fragen verstanden werden, dann ist ein solcher schon von daher kaum zu erwarten. Desweiteren verweist dieses Grundproblem auf »außertheoretische Fragestellungen, die das grundlegende Verhältnis theoretisch-wissenschaftichen Arbeitens zu den gesellschaftlichen Entwicklungen betreffen. Im Rahmen der Regulationstheorie 
geht es dabei insbesondere um den Charakter der Theorie als »Theorie mittlerer Reichweite« (verbunden mit einem deskriptiven Überhang) und um ihr Verhältnis zu den gesellschaftlichen Krisenphänomenen, die ihre Ausformulierung motiviert haben. Diese Fragestellungen können (und müssen) im Rahmen von Theorien zwar reflektiert und bearbeitet werden. Sie liefern aber selbst dann keine unabhängige oder »objektive« theoretische Grundlage, sondern tragen bestenfalls zu einer reflexiven Begriffsbildung bei.

Auf diese Fragestellungen zielen die folgenden Ausführungen ab. Sie schließen dabei insoweit an die Kritik von Scherrer an, als dort einige der wesentlichen Streitpunkte angesprochen und dazu Position bezogen wurde. Neben einer direkten Replik auf einige von ihm angesprochene Punkte, die z.T. das Problem einer Verständigung über divergierende Theoriesprachen hinweg deutlich machen, geht es mir aber vor allem um ein Problem, das bei Scherrer nur am Ende erwähnt wird, das aber zentral für das Verständnis des reflexiven Charakters der Theoriebildung ist: um den Gesellschaftsbegriff und seine theoretischen Grundlagen. Inhaltlich geht es dabei um eine Begründung dafür, was die Rede von einem »Objektivitätsüberhang « gesellschaftlicher Verhältnisse theoretisch bedeutet und warum diese Rede gerade auch heute angebracht und als Moment der Kritik notwendig ist. Ich möchte diese Zuspitzung aber vorweg nicht als »einseitige Parteinahme « für eine der beiden »Analyseebenen von Struktur und Handlung (Scherrer 1995, 458) verstanden wissen. Vielmehr möchte ich seine Kritik dahingehend wenden, daß das von ihm angesprochene Dilemma, nämlich entweder die bekannten Schwächen der Regulationstheorie zu wiederholen oder ihre zentralen Einsichten preiszugeben, gerade aus einer Verleugnung des Vermittlungsproblems resultiert. Dieses Vermittlungsproblem läßt sich aber wiederum erst am Gesellschaftsbegriff angemessen bearbeiten und zielt daher genau auf jenen. Um dieses Argument vorzubereiten, sollen zunächst die beiden anderen Punkte, in denen Scherrer seine Kritik zusammenfaßt, untersucht werden: seine sozialontologischen Annahmen (Verhältnis von Notwendigkeit und Kontingenz) und das Problem der Formanalyse.

\section{Grenzen der Handlungstheorie}

Der Hauptvorwurf von Scherrer gegen unsere, vor allem von Joachim Hirsch und mir in »Politik, Institutionen und Staat « vorgenommene »Kritik der Regulationstheorie« richtet sich gegen die vermeintlich abstrakte Gegenüberstellung von stabilen Akkumulationsphasen und offenen Krisen. Allerdings kann ich weder seiner Kritik noch seinem Gegenmodell, mit 
dem er den festgestellten Mangel überwinden will, so recht folgen und nur vermuten, daß hier ein unterschiedliches Verständnis zentraler Begrifflichkeiten eine ausschlaggebende Rolle spielt. Sein Vorwurf zielt einerseits auf meine Interpretation des Ansatzes von Anthony Giddens. Diese Interpretation mündet in der These, daß es Giddens nicht gelingt, mit dem Begriff der $\gg$ Dualität der Struktur« eine seiner eigenen Problemstellung gemäße konstitutionstheoretische Grundlage zu schaffen und daß vielmehr der Widerspruch zwischen Handlungskompetenzen und Strukturprinzipien unaufgelöst bleibt. Unabhängig von der Angemessenheit dieser Interpretation, die hier nicht diskutiert werden kann, ist damit gerade nicht gesagt, daß das »Fortbestehen des Kapitalismus ... somit wieder rein strukturell, d.h. ohne Bezug auf Präferenzen, Intentionen und Handlungen der Individuen erklärt werden (kann).« (Scherrer 1995, 460) Das genaue Gegenteil ist der Fall: Wäre eine Erklärung ohne den Rückgriff auf die Handlungsdimension möglich, gäbe es den besagten Widerspruch nicht mehr. Er wäre aufgelöst zugunsten des einen Pols, der den Kapitalismus auszeichnenden Strukturprinzipien.

Doch steckt hinter dieser Fehldeutung m.E. eine weitreichende inhaltliche wie terminologische Differenz. Terminologisch tritt sie an den Begriffen des Widerspruchs und der Vermittlung zu Tage: In der Tat wird von mir ein Verständnis von Vermittlung zugrundegelegt, daß nicht die »Überwindung von Gegensätzen « (ebd.) oder die Suche nach den Gegensatz überbrückenden Verbindungsstücken, sondern die Vermittlung durch die Widersprüche hindurch, mithin ein dialektisches Verständnis des Widerspruchsbegriffs meint.' Dieser Vermittlungsbegriff beeinflußt die Begriffskonstruktion wie das Verständnis realgeschichtlicher Prozesse gleichermaBen. Begrifflich ist nochmals daran zu erinnern, daß Struktur und Handlung keineswegs per se Gegensätze oder Dichotomien darstellen, sondern je nach theoretischem Verständnis einander bedingende Dimensionen des Sozialen sind: Ohne im Handeln in Anspruch genommene Regeln ist soziales Handeln im spezifischen Sinn kaum denkbar, weil wir im Grunde dann nicht wissen können, wie eine Handlung fortzusetzen ist (vgl. zum Regel-

1 Anders als Scherrer dies interpretiert sind Institutionen nicht»Verbindungsstücke (ebd.) zwischen gegensätzlichen Polen, sondern sie sind »Erscheinungsformen des Widerspruchs « (Görg 1994b, 72) zwischen verschiedenen Analyscebenen und Realprozessen. Inhaltlich zielt dies auf das Problem der »Embedderness « (s.u.). Un jedoch schon an dieser Stelle kein Mißversäandnis aufkommen zu lassen: ein dialektischer Widerspruch stellt nicht dic von Althusser cntworfene Karikatur dar, eine einfache Einheit entfaltungslogisch und letztlich begriffsmetaphysisch zu denken. (vgl. zun Widerspruchsbegriff: Giddens 1981, 230ff; 1988, 252ff und 432; Görg 1994b, 53ff und 68ff zum hier angesprochonen Vermitthungsproblem) In diesem Sinne schließe ich dabei an den von Jürgen Ritsert (1988) unternommenen Versuch an, die neuere Hegelinterpretation für die Sozialwissenschaften fruchtbar zu machen. 
begriff: Giddens 1988, 69ff). Hierin gründet die von Giddens stark gemachte Ermöglichungsbedingung der Strukturen.

Die Frage ist allerdings, ob ein solches Verständnis von Struktur ausreicht und ob auf in anderen theoretischen Traditionen ausgearbeitete Dimensionen des Strukturbegriffs verzichtet werden kann (vgl. dazu auch: Miiller 1992, 173ff). In dem Versuch von Giddens, diese unterschiedlichen Traditionen und Theorieansätze in einem umfassenden Ansatz im Begriff der »Dualität der Struktur « zu integrieren, lag unser Interesse an seinen Arbeiten begründet. Selbst wenn sein Ansatz diesen Anspruch einlösen könnte, wären damit noch nicht die inhaltlichen Gegensätze, die gesellschaftlichen Entwicklungen betreffend, aufgelöst, die mit den begrifflichen Fragen verbunden aber nicht mit ihnen identisch sind. Doch schon hinsichtlich der Einlösung seines theoretischen Anspruchs melden sich Zweifel, die die unaufgelöste konstitutionstheoretische Spannung betreffen: Auf der einen Seite soll ein konkretes Institutionensystem durch die Handlungskapazitäten der Akteure, durch die Fähigkeit zur rekursiven Selbsteuerung ihres Handelns konstitutiert werden. In diesem Sinne gilt, daß wir in unserem alltäglich-routinisierten Handeln die sozialen Verhältnisse des Kapitalismus reproduzieren (Giddens 1988, 71), daß dieser kein außer uns existierender, sondern ein unserer Subjektivität innewohnender Zusammenhang darstellt. Auf der anderen Seite kennt auch Giddens Reproduktionszusammenhänge, deren Prozeßverlauf genau dieser Steuerung enthoben ist (ebd. 247). Zudem spricht er an manchen Stellen davon, daß konkrete Institutionensysteme durch die den Kapitalismus prägenden Strukturprinzipien konstituiert werden (ebd. 218). Dies scheint mir ein Indiz für das Scheitern seines konstitutionstheoretischen Ansatzes und gleichzeitig ein Ausdruck jenes Objektivitätsüberhangs zu sein, der die kapitalistische Vergesellschafttungsform kennzeichnet, und den es zu begreifen gilt: Ein die Individuen einbegreifender und gleichzeitig ein ihnen fremd gegenüberstehender Zusammenhang zu sein.

Scherrer verwendet in seiner Kritik dagegen eine von E.Laclau und Ch.Mouffe entwickelte Begrifflichkeit, um mit der wechselseitigen Subversion von Notwendigkeit und Kontingenz auf der einen Seite eine vergleichbare, aber um den Widerspruch reduzierte Verhältnisbestimmung zu formulieren, die andererseits die uns unterstellte Dichotomie stabiler Phasen und offener Krisen vermeiden will. Leider setzt er sich jedoch nicht ausreichend mit der von ihm auch erwähnten Kritik an diesem Ansatz auseinander. Er reduziert diese auf die Behauptung, Laclau/Mouffe würden Strukturen nicht richtig berücksichtigen oder einseitig ins Handeln auflösen (Scherrer 1995, 466). Doch ist dies eine etwas einseitige Sichtweise. Krebs/Sablowski $(1992,108)$ bspw. werfen Laclau/Mouffe zu Recht vor, 
die Spezifität kapitalistischer Verhältnisse historisch nicht mehr gegen andere Praxisformen abgrenzen zu können. Dies sei kein zufälliger Fehler sondern einem »Essentialismus des Diskursiven« (ebd.) geschuldet. Darauf geht Scherrer in dieser Form nicht ein, sondern versucht allein zu belegen, daß ihr Ansatz sehr wohl Strukturen kenne, und »das Subjekt« gerade aus der $\gg$ Dislozierung einer Struktur« (Scherrer 1995, 467) resultiere. Doch damit nähert sich seine Ontologie des Sozialen einem »Idealismus der Struktur« (M.Scharping), in dem diese in ihrer Auflösung das Subjekt aus sich entläßt. ${ }^{2}$

Die Begrifflichkeit von Notwendigkeit und Kontingenz führt in ihren ontologischen Grundannahmen unhaltbare Konnotationen mit sich, nämlich die Gleichsetzung von Struktur mit Notwendigkeit und von Handeln (und Subjekt) mit Kontingenz. Durch diese Gleichsetzung werden aber nicht nur problematische Vorannahmen fixiert, sondern auch die eigentlich interessierenden Fragen ausgeklammert. Nicht nur ist Notwendigkeit im strengen Sinn dem Sozialen gerade äußerlich: »Strukturen, d.h. Gesetze gesellschaftlicher Praxis, die Wiederholungen steuern« (Scherrer 1995,464), tun dies niemals mit Notwendigkeit in dem Sinn, daß soziale Akteure zu ganz bestimmten Handlungen gezwungen und insofern in ihrem Handeln determiniert würden (von einem Verständnis von Strukturen, die als Ermöglichungsbedingungen für Handeln dienen, hier ganz abgesehen). In Strukturen sind Notwendigkeit und Kontingenz vielmehr immer miteinander verbunden: Das Warenverhältnis stellt nur insofern eine Notwendigkeit dar, als es uns die Freiheit des Konsumenten gibt; der Staatsbürger ist (in der Demokratie) nur insofern auf die Staatsform verpflichtet, als er die Wahl hat; usw.

Die Existenz von Gesetzen, die nicht noch durch die Selbsteuerung der Handelnden vermittelt sind, lehnt Giddens mit guten Gründen ab (Giddens 1988, 402ff). Deswegen ist auch umgekehrt nicht die Kontingenz des Handelns das Problem, denn diese ist dem Sozialen eben immer inhärent: Bis auf vielleicht die Ausnahmesituation der totalen Institution behalten Akteure immer verschiedene Handlungsmöglichkeiten und die Fähigkeit zur rekursiven Transformation der Handlungsmuster, in denen sie sich bewegen. Sie behalten selbst als Teilnehmer institutionalisierter Praxisformen immer noch Handlungsoptionen, die weder von einem (sozialwissenschaftlichen) Beobachter noch von einem anderen Akteur jemals völlig antizipiert werden können. ${ }^{3}$ Das Problem ist also nicht, daß sich auflösende Strukturen

2 Individuelle Selbstbestimmung als mmißlungene strukturelle Identität (Laclau, zit. nach Scherrer 1995, 465, Herv. i.O.) zu bezeichnen, nivelliert sowohl die Fähigkeit zur Entgegensetzung als auch deren potentielle Einschränkung in der mißlingenden Identität.

3 Das Problem besteht also darin, Regelmäßigkeiten des sozialen Handelns zu erklären, ohne auf eine der beiden traditionellen Optionen zurückzugreifen, die von Giddens beide als 
Kontingenzen hinterlassen. Das Problem ist (in Giddensscher Terminologie) die »Dialektik der Herrschaft « innerhalb bestehender Strukturen. Genauso wie Strukturen nicht unbedingt Notwendigkeiten darstellen, ist Kontingenz nicht mit Selbstbestimmung gegen Formen der Herrschaft identisch. Strukturen sind genauso als herrschaftlich geprägte erst zu identifizieren und zu analysieren ${ }^{4}$, wie umgekehrt aufzuzeigen ist, daß bestimmte Handlungsformen über die in den strukturellen Mustern angelegten Handlungsoptionen hinausreichen. $\mathrm{Zu}$ zeigen wäre, daß Formen der Selbstbestimmung nicht nur die ihnen gewährten Freiheiten (des Konsumenten, des Staatsbürgers, aber auch die kleinen Fluchten des Alltags zur Erleichterung der Subalternität) nutzen, sondern auf die Veränderung der institutionellen Formen abzielen.

Auf die Regulationstheorie gewendet heißt dies: Handlungsmöglichkeiten entstehen keineswegs erst in der Krise. Abgesehen von den Optionen, die selbst stabile Institutionen immer auch enthalten und die immer auch reale Freiheitsspielräume sind, können mindestens drei verschiedene darüberhinaus gehende Gestaltungsmöglichkeiten theoretisch unterschieden werden: Gestaltungsprozesse, die auf die fortwährende Reorganisation institutioneller Konfigurationen gerichtet sind, ohne daß diese selbst in der Krise wären (institutionelles Handeln), von solchen, die die Etablierung neuer institutioneller Arrangements angesichts des Versagens oder der Unangemessenheit der bestehenden intendieren (transformierendes Handeln) und letztlich solchen, die auf die Transformation der den Kapitalismus kennzeichnenden Strukturprinzipien gerichtet sind (strukturtransformierendes Handeln; vgl, zu den Begriffen: Esser/Görg/Hirsch/Sablowski 1994, 220). Diese letzte Gestaltungsoption muß keineswegs auf Krisensituationen beschränkt sein. Vielleicht lag darin sogar der entscheidende Fehler der klassischen Revolutionstheorie, die den »großen Kladderadatsch« und die revolutionäre Überwindung des Kapitalismus immer wenn auch in verschie-

unzureichend und als ideologieverdächtig kritisiert werden: individualistische Vertragstheorien (wie im Anschluß 3 an Hobbes) vs normativistische Integrationstheorien (a la Parsons). Das nun keineswegs neve Problem besteht dann darin, von einem in und durch Institutionen vergesellschaftetem Subjekt auszugehen, das gleichwohl die Fähigkeit zur Selbstbestimmung gegen diese Institutionen hat, ohne daß diese Fähigkeit, da hat Scherrer (vgl. 1995, 465) völlig recht, in einem vermeintlichen »Wesen « dieses Subjekts garantiert ist: Jeder Akteur, das einzelne Individuum wie in noch größerem Maße alle Kollektive, sind zunächst gesellschaftlich bestimmt.

4 Ein keineswegs triviales Problem, denn daran scheitert in letzter Instanz auch Giddens mit seiner Ontologisierung der $\gg$ Dialektik der Herrschaft«. Aufgrund der Vermischung nit dem Machtbegriff tendiert diese dazu, verschiedene Ausprägungen von Herrschaft nicht mehr konkret unterscheiden zu können (Görg 1994b, 45f). Im Unterschied zu Scherrer wïrde ich auch darauf beharren, daß die Frage nach Selbstbestimmung die Frage nach Gründen und Motiven der Handelnden einschließt und daher sowohl theoretisch-methodisch als auch politisch bestimmte Herangehensweisen an diese Motive erforderlich sind. 
dener Weise verbunden hatte. Wir haben dagegen für diesen letzten Gestaltungsprozeß den Begriff des »radikalen Reformismus « zu entwickeln versucht. Auf seine politische wie theoretische Bedeutung wird noch einzugehen sein.

Aber die ersten beiden Gestaltungsdimensionen sind, nur weil ihre jeweilige Reichweite die Strukturprinzipien des Kapitalismus nicht überschreitet, deshalb nicht weniger (Re)Strukturierungsprozesse des Institutionensystems. Eine gewisse Offenheit ist dem sozialen Prozeß aber nicht nur aus abstrakten theoretischen Überlegungen zuzugestehen, sondern auch aus der Erkenntnis heraus, daß tragende institutionelle Konfigurationen niemals statisch sind, selbst als gültige Orientierungsmuster sozialen Kämpfen immer nur ein kompromißhaft stabilisiertes Terrain vorgeben, das sich gerade durch die weiteren Kämpfe entwickelt und damit auch die Kräfteverhältnisse zwischen den verschiedenen Akteuren verändern kann. Trotzdem geht mir die Formulierung zu weit, daß die »Entwicklungsrichtung eines Akkumulationsregimes ... jederzeit latent offen (ist) « Scherrer 1995, 480). »Latent offen « oder »Jederzeit «? Letzteres würde einen Voluntarismus darstellen, der nur schwer mit dem oben zitierten Strukturbegriff zusammengeht. Latent offen sind Akkumulationsregime und Regulationsweisen allerdings in mehrfacher Hinsicht: Ihre Entwicklungen sind sozialwissenschaftlich niemals genau zu prognostizieren, sie lassen wohl immer mehrere Optionen ihrer Weiterentwicklung gemäß den Handlungsstrategien der Akteure zu (keine Notwendigkeit) und ihre Kohärenz kann durch diese Reorganisation beeinträchtigt werden. Ein transformierendes Handeln ist nicht erst auf den Ausbruch einer Krise des Institutionensystems verwiesen, sondern kann diese sogar erst aktiv herbeiführen. Nur insofern sind also Krisen gegenüber stabilen Akkumulationsphasen wirklich »offener«, als sie den auch in den stabilen Phasen enthaltenen Widerspruch zwischen institutionalisierten Handlungsformen und darüber hinausgehenden Handlungskompetenzen offenlegen.

Die wechselseitige Subversion von Notwendigkeit und Kontingenz unterläuft aber nicht nur die $\gg$ Dialektik der Herrschaft $\ll$, sie ist, wie Scherrer selbst feststellt, auch »zu arm an Bedeutung ..., um die wirklichen sozialen Kämpfe und Praxen zu analysieren « (Schiesser, zit. nach Scherrer 1995, 467). Bezogen auf den Institutionenbegriff gilt dies in einem spezifischen

5 Ob sie damit auch real die Chance einer weitergehenden Gestaltung sozialer Verhältnisse bieten, ist eine andere Frage und nur gemäß den vorhandenen Interessen, Machtverhältnissen und politischen Strategien zu entscheiden (Görg 1994b, 65f). Das heißt z.B. in der Bundesrepublik heute ganz konkret: Gestaltung erschöpft sich in der vermeintlichen Exekution von Sachzwängen der Weltmarktkonkurrenz, also gerade in der Anpassung an strukturelle Bedingungen. 
Sinne. Stärker noch als ihr Beitrag zur Aufklärung der Dialektik des Handelns liegt die Leistung der Giddensschen Sozialtheorie für den Institutionenbegriff gerade in seiner horizontalen Vielschichtigkeit, die er durch die Verknüpfung unterschiedlicher Formen regelgeleiteten (d.h. an gemeinsamen »Tiefencodes « orientiert) und ressourcenorientierten Handelns erreicht. Diese Verknüpfung könnte, würde sie über die Grundlegung bei Giddens hinaus fruchtbar gemacht, eine ganze Reihe sozialwissenschaftlicher Einseitigkeiten überwinden helfen. Sie zielt nämlich auf die Verbindung diskursiver Praxisformen nicht nur mit normativen Elementen, sondern vor allem auf das Verhältnis beider zu ungleich verteilten allokativen und autoritativen Ressourcen. Sie vermeidet die Einseitigkeit, angesichts der Anerkennung des »Eigensinns des Sozialens « sich auf normative und/oder diskursive Synthesisformen zu beschränken und auf die Analyse strukturierter Ungleichheit (Klassen) oder aggregierter Macht- und Herrschaftsverhältnisse (Staat) zu verzichten. Institutionen sind dagegen immer in der Verbindung dieser Handlungsdimensionen zu analysieren und je nach ihrer konkreten Ausprägung zu unterscheiden. ${ }^{6}$ Die Vorteile dieser Vorgehensweise wären am konkreten Material, bspw. am Zusammenspiel von normativen und diskursiven Elementen mit Machtverhältnissen und spezifischen Akkumulationsstrategien im Rahmen bestimmter Managementstrategien zu untersuchen. Nach wie vor leiden dementsprechende Untersuchungen der Regulationstheorie nämlich am theoretisch unausgewiesenen Verhältnis ökonomischer und im weiteren Sinne sozialer Dimensionen (vgl. schon Mahnkopf 1988).

Trotz dieser Vorteile, die der Giddenssche Institutionenbegriff für die Analyse historischer Phasen mit sich bringt, liegt das eigentliche Problem des Vermittlungsbegriffs jedoch auf einer anderen Ebene, die nicht mit der »Dialektik der Herrschaft « identisch ist. Würde man bei diesen Analyseebenen stehenbleiben wären soziale Synthesis und sozialer Wandel aus dem Handeln der Akteure heraus vollständig zu erklären. Wie kommt es aber, $\mathrm{da} B$ die strategische Analyse bei Giddens zur Erklärung geschichtlicher Prozesse nicht ausreicht, so daß wir sie durch eine strukturelle Analyse ergänzen müssen? ( $V$ gl. dazu Görg 1994b, 34ff). Auf diese Notwendigkeit verweist auch Scherrer in der Kritik an Sablowski mit Blick auf die Eigendynamik konjunktureller Prozesse und auf funktionale Zwänge (Scherrer 1995, 461 und 475). Doch darf dieser Hinweis sich nicht mit der Existenz dieser Eigendynamik oder dem Bestehen funktionaler Zusam-

6 Hier komnt die Weberianische Tradition in Giddens zum Vorschein: kapitalistische Ökonomie und Staat ruhen auf kulturellen Bedeutungen auf. Andererseits läBt sich soziale Synthesis nicht auf normative oder symbolische Handlungsformen reduzieren, sondern hat sich ihnen gegenüber verselbständigt; vgl. unten. 
menhänge, die den Akteuren gegenüber als mehr oder weniger undurchschaute bzw. unveränderbare Zusammenhänge auftreten, zufrieden geben. $\mathrm{Zu}$ erklären ist, wie es dazu kommt, daß soziale Zusammenhänge den sozialen Akteuren als funktionale Zusammenhänge entgegentreten; wie es zu verstehen ist, daß diese Verhältnisse ihrem Handeln partiell entzogen sind und diesem als fremde und undurchschaubare Mächte erscheinen - obwohl die Akteure sie doch in ihrem Handeln reproduzieren. Das Vermittlungsproblem läßt sich daher so formulieren: Was sind die Merkmale sozialer Verhältnisse, die diese Verselbständigung aus dem Handeln heraus und ihm entgegengesetzt erklärbar machen können? An dieser Stelle treten die Grenzen der Handlungstheorie zum Vorschein, die allerdings nicht einfach konstatiert noch unter Verweis auf die Komplexität moderner Gesellschaften sozialontologisch naturalisiert werden sollen, sondern die mit dem Begriff der sozialen Form bearbeiten werden können.

\section{Antagonistische Vergesellschaftung und soxiale Form}

Im Kern des Vermittlungsproblems steht also die Frage, wie es zu erklären ist, daß sich spezifische soziale Verhältnisse gegen die in ihnen handelnden Akteure verselbständigen, obwohl ihre Reproduktion vom Handeln eben dieser Akteure in gewisser Weise abhängig ist. »In gewisser Weise《 heißt dabei zunächst: Es bestehen funktionale Zusammenhänge (bspw. im Sinne von Reproduktionskreisläufen), die nicht reflexiv gesteuert, wohl aber erst durch das kompetente soziale Handeln der Akteure hindurch aufrecht erhalten werden. ${ }^{7}$ Das Vermittlungsproblem ist damit kein Problem logischer Übergänge (Scherrer 1995, 469), sondern zielt auf die zentrale Aussage der Regulationstheorie, daß sich kapitalistische Verhältnisse nur durch institutionelle Formen hindurch stabilisieren können, daß es funktionale Zwänge gibt, die aber stabilisiert werden müssen. Damit steht aber der Status der Wertheorie zur Debatte - und ebenfalls unterschiedliche Rezeptionsweisen der Marxschen Theorie.

Die Probleme, die sich durch die unterschiedlichen Weisen der theoretischen Aneignung der Marxschen Theorie stellen, sind nicht zu unterschätzen und an dieser Stelle auch nicht zu bewältigen. Die zentrale Frage ist

7 In dieser Formulierung sind gravierende methodologische und »sozialontologische Pro- $^{-}$ bleme versteckt, die an dieser Stelle weder ausgebreitet noch gelöst werden können. In der Tat ist hier das Problem der Privilegierung eines spezifischen theoretischen Zugangs zur Erkenntnis dieser Zusammenhänge und seiner Unterscheidung vom »common sense« (Giddens) der Alltagsakteure angelegt. Hier gibt es sehr unterschiedliche Antwortstrategien, die sich heute vor allem an der Frage reiben, ob nicht diese Unterscheidung selbst das Problem sei und wissenschaftskritisch destruiert/dekonstruiert werden müsse. Im folgenden wird eine Lösung anvisiert, die dieses Problem dagegen zur Grundlage des kritischen Potentials der Theorie macht (ähnlich auch Giddens 1988, 405ff). 
dabei der Umgang mit dem »Althusserschen Erbe« (Lipietz), wobei diese Diskussion durch die neuerliche Althusser-Rezeption, die ganz andere Schwerpunkte setzt als die Diskussion in den 70er Jahren, noch zusätzlich verkompliziert wird. Bekanntermaßen scheiden sich aber am Status der Wertformanalyse, d.h. der Marxschen Begründungsstrategie für die Werttheorie, auch die von ihm inspirierten Geister. Für die einen ist sie hegelianisierender metaphysischer Unfug, für die anderen der Kern der Marxschen Ökonomiekritik überhaupt. Dieser Streit kann hier weder geschlichtet noch einfach umgangen werden. Da unsere Rekonstruktion der gesellschaftstheoretischen Grundlagen der Regulationstheorie auf der Marxschen Formanalyse aufbaut, müssen wenigstens einige Hauptpunkte der Kontroverse kurz resümmiert werden.

Es ist keineswegs so, daß die Werttheorie auf restriktiven Modellannahmen beruht, die an der komplexen Wirklichkeit scheitern (so Scherrer 1995, 468), weil sie das $\gg$ Modell des reinen Kapitalismus« (ebd. 469) ausmale. Scherrer unterschlägt hier einfach die Differenz zwischen »bürgerlicher« Ökonomietheorie und Marxscher Kritik, die nämlich gerade auf die Begründung der Kategorien abzielt. Und er ignoriert dabei eben das Kernstück der Kritik der politischen Ökonomie, die Wertformanalyse. In dieser wird von Marx kein idealisiertes Modell sozialer Tauschprozesse entworfen, sondern im Gegenteil die sozialen Implikationen diskutiert, auf die die basalen ökonomischen Kategorien von Wert und Preis ihrerseits erst aufruhen. Diese werden aber von der klassischen wie neoklassischen Ökonomie immer vorausgesetzt, ohne daß in ihrem kategorialen Rahmen jemals eine Klärung dieser Voraussetzungen erfolgt wäre (Backhaus 1996). Der entscheidende Vorteil der Werttheorie liegt also gerade darin, daß sie die Abstraktionen, die die ökonomischen Kategorien der Nationalökonomie beinhalten, als soziale Prozesse verstehbar macht. Inhaltlich zeigt die Formanalyse dabei auf, daß spezifische Widersprïche und Antagonismen der formalen Einheit des gesellschaftlichen Reproduktionsprozesses schon immanent sind, daß dieser also keineswegs auf funktionalen Erfordernissen gesellschaftlicher Synthesis beruht, sondern vielmehr ein Klassen- und Ausbeutungsverhältnis einschließt und in sich von einer grundlegenden Irrationalität und Krisenhaftigkeit durchzogen ist (Görg 1994c).

In der Kritik von Scherrer werden diese Grundaussagen der Werttheorie dagegen mehr oder weniger explizit negiert: Weder die Existenz eines sozialen Antagonismus noch ein Widerspruch im gesellschaftlichen Charakter der Warenproduktion wird von ihm unter Berufung auf Laclau gelten gelassen (Scherrer 1995, 472). Trotzdem will er am Ende doch die Formanalyse verteidigen, die geeignet sei, »auf bestimmte funktionale Zusammenhänge bzw. Inkompatibilitäten aufmerksam (zu) machen.« (ebd. 479). 
Nun sind in der Tat Widersprüche und Antagonismen nicht identisch mit Konflikten auf der Handlungsebene. Aber die von Scherrer vorgenommene Reduktion der Formanalyse unterschlägt gerade deren Pointe, daß nicht nur funktionale Zusammenhänge benannt, sondern deren Einbettung in wie umgekehrt ihre Verselbständigung von sozialen Verhältnissen erklärt werden soll. In diesem Erklärungsanspruch unterscheidet sie sich von Ansätzen zu einer institutionalistischen Ökonomie, die wie Polanyi die gleiche Widersprüchlichkeit zwei gegensätzlichen Organisationsprinzipien zuspricht (Polanyi 1977, 172). Verselbständigung ist bei Marx eben nicht wie bei Polanyi Ergebnis des selbstregulierenden Marktes, von dessen selbstund gesellschaftsdestruktivem Charakter dieser überzeugt war (ebd. 103). Insofern ist die »Hochzeit von Marxismus und Institutionalismus (Elam 1994) nicht ganz so unproblematisch. Statt von Selbstregulierung wäre von den sozialen Implikationen zu sprechen, auf denen die sozialen Formen gerade aufbauen: Die Arbeitskraft ist eben nicht nur eine unechte Ware, wie dies Polanyi in kritischer Absicht betont. Diese Eigenschaft (bzw. die Differenz zwischen Arbeiter und Arbeitskraft) ist vielmehr gerade die Voraussetzung dafür, daß sie als Quelle des Mehrwerts dient. Insofern stellt das Lohnverhältnis auch eines der zentralen Strukturprinzipien kapitalistischer Vergesellschaftung dar, daß bei aller institutionellen Ausformung auch reproduziert werden $» m u ß \ll:$ Ohne es gibt es weder Kapital noch Akkumulation. Trotzdem machen die regulationstheoretischen Arbeiten gerade auch deutlich, daß seine institutionelle Einbettung nicht nur neue Möglicheiten der Kapitalverwertung eröffnet hat (Komsumgütermarkt), sondern auch der selbstdestruktive Mechanismus nur in modifizierter Form gilt. Nur als nichtkorrigierter würde auch nach Polanyi der Marktmeachismus »zur Zerstörung der Gesellschaft führen.« (Polanyi 100) Solange aber diese Korrektur durch die institutionelle Einbettung gegeben ist, verlagert sich das Problem auf die Frage, wie sich der Widerspruch zwischen Einbettung und Verselbständigung historisch entwickelt. Ein konkretes Beispiel wäre heute die Rolle neoliberaler Politikmodelle, die sich für die ungehinderte Freisetzung dieses zerstörerischen Organisationsprinzips einsetzen und insofern auch nicht zu stabilen Regulationsweisen führen (Hirsch 1995).

Im Begriff der sozialen Form ist also ein entscheidender Gegensatz zu institutionalistischen Ansätzen angelegt, der mit dem Begriff des strukturellen Widerspruchs zusammenhängt. Wir hatten im Anschluß an Giddens hervorgehoben, daß Widersprüche nicht nur Dysfunktionalitäten in einem Reproduktionszusammenhang sind, sondern eine spezifische Relation wesentlicher struktureller Merkmale dieses Zusammenhangs beinhalten: einen Gegensatz von Strukturprinzipien, in dem deren wechselseitiger Ausschluß und ihre Verwiesenheit aufeinander gerade zusammengehören (Görg 
1994b, 53). Giddens (1981, 233) nennt als Beispiel für seinen Widerspruchsbegriff den Widerspruch zwischen privater Aneignung und dem gesellschaftlichem Charakter der Produktion. Entscheidend ist nun, hier stärker als Giddens selbst auf die Form zu verweisen, in dem dieser Widerspruch erscheint, nämlich eben auf die Wertform und den in sie eingehenden Widerspruch von Gebrauchswert und Tauschwert. Die Wertformanalyse ermöglicht es nämlich, die Nicht-Einheitlichkeit eines Gesamtzusammenhangs gesellschaftlicher Einheit zu denken. Deutlicher als bei Marx wäre zudem der »Widerspruch der bürgerlichen Konstitution« und die Staatsform zu berücksichtigen, die eben nicht ein aus ökonomischen Verhältnissen abgeleiteter sondern ein die grundlegenden sozialen Verhältnisse konstitutiv prägender Widerspruch darstellt (Hirsch 1994).

Der Wert stellt die innere Einheit eines Reproduktionsprozesses dar, der von den Akteuren nicht reflexiv gesteuert wird, der sich aber nicht wirklich selbst reproduzieren kann, sondern auf die handelnden Akteure gerade angewiesen bleibt. Gegen Marx Annahme ist nämlich nicht einfach davon auszugehen, daß die Bereitschaft der Akteure, als »Hüter die Waren zu Markte zu tragen (MEW 23, 99) und als »Charaktermasken« (ebd., 100) der ökonomischen Verhältnisse zu fungieren, historisch einfach unterstellt werden kann. Vielmehr ist in den kapitalistischen Reproduktionsbedingungen strukturell ein Widerspruch angelegt, der für den Prozeß der Verselbständigung grundlegend ist. Mit dem Begriff der Wertform (und den ihr korrespondierenden Begriffen von Geld und Kapital) wird von Marx eine Synthesisform paradigmatisch analysiert, die nicht eine universelle Verdinglichung aller sozialen Verhältnisse unterstellt, sondern diesen Prozeß der Verselbständigung erfaßt. Soziale Formen machen strukturelle Widersprüche prozessierbar, sind die Bewegungs»form《 dieser Widersprüche, stehen aber selbst wieder im Widerspruch zu den sozialen Voraussetzungen auf der Handlungsseite, auf denen ihre eigenen Funktionsgesetzlichkeiten aufbauen. Deswegen geht es beim Formbegriff auch nicht um einen ideologiekritischen Generalverdacht gegenüber dem Altagswissen sozialer Akteure, selbst wenn er fetischisierte Verhältnisse auf den Begriff bringt. Und genausowenig kann an dem zuweilen formulierten Anspruch festgehalten werden, Gesellschaft als expressive Totalität aus einem »Kern«, der Waren- und Wertform abzuleiten, d.h. die Komplexität des historischgesellschaftlichen Prozesses auf diesen Kern kausal zurückzuführen (vgl. Ritsert 1973). Die Annahme einer linearen Entfaltung eines »Kerns« kapitalistischer Vergesellschaftung ist unserer Ansicht nach nicht haltbar - weder negativ (Zusammenbruch oder universale Verdinglichung) noch positiv (endgültige reformistische Bändigung und Transformation). Zentral ist die in der Regulationstheorie erforschte Einheit von Kontinuität und Diskonti- 
nuität, allerdings als Einheit von Reproduktion und Regulation. Regulation ist, wie oben beim Lohnverhältnis angedeutet, immer auch Reproduktion strukturell verankerter Positionen und daraus sich ergebender (Geld- oder Macht-)Ressourcen im Rahmen kapitalistischer Verhältnisse, und deren Reproduktion schließt immer eine institutionelle Regulation ein.

\section{Gesellschaft als Totalität}

Auch wenn die Ausführungen zum Begriff der sozialen Form den Charme einer etwas verknöcherten Marxschen Orthodoxie ausstrahlen dürften - mit ihnen soll keineswegs der Eindruck erweckt werden, in der Marxschen Theorie liege die Lösung der uns beschäftigenden Probleme schon vorgezeichnet und müsse nur neu entdeckt werden. Der Formbegriff kann lediglich an einer präzisen Stelle gegenüber konkurrierenden gesellschaftstheoretischen Ansätzen neue (alte) Akzente setzen und dabei spezifische Vorteile geltend machen. Wo heute die Alternative unausweichlich scheint, entweder auf gesellschaftstheoretische Fragestellungen zu verzichten oder zu systemtheoretischen Denkweisen Zuflucht zu nehmen, bietet er die Möglichkeit, an den Intentionen einer kritischen Gesellschaftstheorie festzuhalten. Wenn Gesellschaftstheorie ganz allgemein gesagt der Versuch ist, einen Zusammenhang herzustellen, in den einzelne Entwicklungen eingelassen sind, dann ist heute durchaus strittig, ob dies noch eine sinnvolle Perspektive ist. Christoph Scherrer $(1995,479)$ jedenfalls glaubt, auf diese Perspektive und insbesondere auch auf die darin eingeschlossene staatstheoretische Fundierung verzichten zu können. Gegen diesen Verzicht sollen zwei Argumente geltend gemacht werden: (1) Allein auf gesellschaftstheoretischer Ebene kann die oben angedeutete Grundproblematik der Regulationstheorie, die Einheit von Reproduktion und Regulation zu denken, wenn nicht wirklich gelöst so jedoch einem Verständnis näher gebracht werden. (2) Werden Akkumulationsprozesse unter Ausblendung ihrer gesellschaftlichen (nicht-ökonomischen) Voraussetzungen analysiert (so verstehe ich die Intention von Scherrer), dann wird der Schein der Selbstgenügsamkeit und Alternativlosigkeit dieser Prozesse theoretisch noch verstärkt, während es darauf ankäme, ihre herrschaftliche Verfaßtheit und ihre strukturelle Veränderbarkeit theoretisch aufzuzeigen.

(1) Die Grundproblematik der Reglationstheorie läßt sich auf sehr verschiedene Weise beschreiben. Hier wurde vor allem herausgesiellt, daß die Regulation in spezifischen institutionellen Formen (Embeddedness) zumindest bislang gleichzeitig eine Reproduktion struktureller Merkmale kapitalistischer Verhältnisse impliziert (soziale Formen). In Erscheinung tritt dieses Problem bei der Untersuchung des Übergangs zwischen zwei 
geschichtlichen Phasen. Hier spielen zwei Sachverhalte herein, die beide für sich relativ unstrittig, deren genaueres Verständnis nach wie vor allerdings unklar ist. Denn ein Übergang ist genauso ein Ergebnis sozialer Kämpfe wie die jeweils neue institutionelle Konfiguration in der Formulierung von Lipietz eine historische »Fundsache « ist. Ausgespart bleibt in dieser Lösung allerdings die Frage, warum denn die »Fundsache« bestimmte Verhälinisse eben auch reproduziert. Hier scheinen zwei Strategien des Umgangs mit diesem Problem vorzuherrschen: Entweder die Ausklammerung oder die Leugnung des Problems. Ausgeklammert wird das Problem bei Scherrer $(1995,480$ ), der die Fortdauer der $\gg$ Hegemonialstruktur kapitalistischer Gesellschaften als gegeben voraussetzt. « Diese Voraussetzung ist aber hochgradig erklärungsbedürftig. Konsequenter sind dagegen die Strategien der Leugnung, die die »Fundsachen« allein als Ergebnis einer hegemonialen Stabilisierung ohne Berücksichtung eigenständiger struktureller Komponenten erklären wollen (vgl. Krebs/Sablowski 1992, Sablowski 1994). Hier stellt sich dann das oben diskutierte Problem, ob auf funktionale Zusammenhänge und konjunkturelle Verlaufsgesetzlichkeiten als Erklärungsmomente verzichtet werden kann.

Es lohnt sich, hier nochmals kurz auf die theoriegeschichtlichen Voraussetzungen der Formulierung von Lipietz einzugehen. Denn diese hat ihre Wurzeln in der Schwierigkeit des strukturalen Marxismus von Althusser gesellschaftliche Veränderung zu denken. Wie Lipietz herausgearbeitet hat, sind es gerade die mit dem Gedanken an geschichtliche Veränderungen verbundenen Elemente des Widerspruchs und des Subjekts, in denen sich die Regulationstheoretiker als »Rebellensöhne« Althussers von diesem unterscheiden (Lipietz 1992). Die Formulierung des »glücklichen Fundes « wird jedoch von Lipietz gerade aus einem Theoriefragment Althussers abgeleitet: aus der Theorie der Übergänge (ebd., 28ff). Hier aber steckt der Kern des Problems: Denn wenn alle Elemente einer Regulationsweise in ihrem konkreten Zusammenspiel relational bestimmt sind, dann wird der Übergang zwischen zwei in dieser Weise bestimmten Phasen zur »black box«. Anders ausgedrückt: Um die historische Diskontinuität wirklich erklären zu können, müßte nicht nur die Althussersche Ontologie, sondern ebenso seine Methodologie aufgeben werden (anders dagegen Lipietz, ebd. 41; vgl. dazu Görg 1994a, bes. 22ff).

Wir hatten das hier ungelöste Problem im Anschluß an Giddens mit dem Begriff des »strukturellen Zwangs « zu bearbeiten und die dabei auftretenden methodologischen und inhaltlichen Folgeprobleme zu bestimmen versucht (vgl. Görg 1994b, 61ff). Dabei ist nicht unterstellt, daß kapitalistische Strukturprinzipien die Ausgestaltung einer neuen Phase vollständig determinieren, was nach dem oben gesagten ausgeschlossen ist. Vielmehr 
üben sie einen Zwang insoweit aus, als spezifische Verhältnisse einerseits in die routinisierte Praxis der Akteure eingewandert sind, also diese Gewohnheiten immanent strukturieren und damit bestimmte Handlungsformen (z.B. utilitaristische Handlungsmuster gegenüber anderen) privilegieren. Andererseits stehen sie als »geronnene Geschichte « den Akteuren gegenüber: nicht nur in Form angehäufter Machtressourcen oder in Form von Kapital, sondern auch in den strukturellen Formen sozialer Reproduktion. Einen strukturellen Zwang üben sie dabei insofern aus, als sie nicht nur direkt Handlungsoptionen eröffnen bzw. verschließen, sondern darüber hinaus die rekursiven Handlungskompetenzen der Akteure einschränken. Ein struktureller Zwang liegt also im Zusammenspiel der beiden Aspekte begründet: $\mathrm{Da} \beta$ bestimmte Handlungsformen strukturell verankerten Machtverhältnissen dienen. Warum also bestimmte Handlungsformen sich durchhalten oder verfestigen, ist nicht im Rückgriff auf die unmittelbaren Strategien allein zu beantworten, sondern es bedarf dazu des Blicks auf die Strukturprinzipien. Warum bspw. trotz aller fehlgeschlagenen Erfahrungen dieses Jahrhunderts grundsätzliche gesellschaftliche Änderungen immer noch vom Staat erwartet werden, das läßt sich erst mit Hilfe eines »Staatsfetischs « (J.Hirsch) erklären, der seinen Grund in der Staatsform hat.

(2) Diese Bemerkungen sind noch nicht ausreichend, um eine »Theorie der Übergänge« auf andere Gundlagen zu stellen. Vielmehr soll gezeigt werden, daß die Einsichten der Regulationstheorie nur bewahrt werden können, wenn das Vermittlungsproblem im Sinne einer Verhältnisbestimmung von strukturellen Zwang und rekursiver Selbsteuerung des Handeln ernst genommen wird. Dieses Verhältnis ist unter kapitalistischen Bedingungen aufgrund der spezifischen sozialen Formen ein widersprüchliches Verhältnis, das nun allerdings in empirisch sehr unterschiedlichen Ausdrucksformen erscheinen kann. Aufgabe einer kritischen Gesellschaftstheorie wäre es, diese Widersprüchlichkeit sozialer Verhältnisse zu bearbeiten, was zweierlei meint: In der Tradition dialektischer Gesellschaftstheorie, insbesondere im Anschluß an Adorno (1979, 9ff), bezeichnet der Gesellschaftsbegriff einerseits einen Funktionszusammenhang, der den sozialen Akteuren als außer ihnen liegender Zwangsmechanismus erscheint. Andererseits sollte in der Kritik dieser Zusammenhang in der Weise »auf den Begriff gebracht werden«, daß seine vermeintliche Funktionalität destruiert, d.h. sein Herrschaftscharakter und seine Veränderbarkeit sichtbar wird. In diese Kontruktion ist eine Differenz zwischen Funktionszusammenhang einerseits und Funktionalität bestimmter Einzelprozesse andererseits eingelassen, die weiter herauszuarbeiten wäre.

Der kritische Sinn des Gesellschaftsbegiffs liegt dann in der Destruktion der vermeintlichen Funktionalität eines Zusammenhang, der sich gleich- 
wohl repressiv zur Geltung bringt. Die Repressivität des Zusammenhangs, unabhängig von weiteren spezifischen Herrschaftsverhältnissen, ist gerade in der Einschränkung der Handlungskompetenzen sozialer Akteure angelegt. Ohne diese Einschränkung ließe sich die vermeintliche Funktionalität kaum von der systemtheoretischen Denkfigur unterscheiden, daß die modernen Gesellschaften zur Bearbeitung ihrer gestiegenen Komplexität funktionale Subsysteme der Kleinarbeitung dieser Komplexität entwickelt hätten - eine Denkweise, die die Verselbständigung gesellschaftlicher $\mathrm{Zu}$ sammenhänge den Akteuren gegenüber zum Merkmal der Moderne naturalisiert.

Wird in der Systemtheorie unterschiedlicher Spielarten der herrschaftliche Charakter des sozialen Prozesses unterschlagen, dann gibt es allerdings auf der Gegenseite auch den umgekehrten Versuch, noch die Destruktion des vermeintlich funktionalen Zusammenhangs ideologietheoretisch zu unterlaufen. Denn wenn, wie dies Alex Demirovic in seiner Rekonstruktion der Regulationstheorie unternimmt, noch die Begrifflichkeiten, mit denen soziale Prozesse analysiert werden, selbst als Teil des regulativen Gesamtzusammenhangs interpretiert werden, dann löst sich in der permanent verändernden Praxis sozialer Auseinandersetzungen auch das Geheimnis auf, das seiner Ansicht nach »die Regulationisten ständig lüften wollen, was nämlich die antagonistische Gesellschaft eigentlich zusammenhält.« (Demirovic 1992, 153) In dieser Strategie sind es die kompetenten Akteure, die die sozialen Verhältnisse willentlich und wissentlich reproduzieren. Anders als Scherrer würde ich dahinter nicht per se den Voluntarismus wittern (Scherrer 1995, 476), denn die sozialen Entwicklungen ergeben sich auch hier erst im Zusammenspiel vieler Akteure, von denen niemand nach belieben den Gang der Dinge bestimmen kann.

Das Problem liegt vielmehr im genauen Verständnis der Kompetenz, die im Handeln zum Ausdruck kommt. Ohne Frage sind wir kompetente Akteure unseres Alltagsleben in dem Sinne, daß wir (in der Regel) wissen, was wir tun, und dies auch im Prinzip wenigstens wollen. Die Frage ist aber, ob wir die Funktionsweise kapitalistischer Vergesellschaftung dadurch verstehen lernen, daß wir diese Handlungen und die ihnen zugrundeliegenden Motive und Absichten analysieren. Demirovic möchte den bei Gramsci angelegten, aber durchaus bei Adorno in Ansätzen vorhandenen kritischen Grundgedanken radikalisieren, daß soziale Verhältnisse immer diskursiv oder geistig vermittelt sind und durch die bewußte Praxis der Akteure hindurch reproduziert werden. Gegen Scherrer enthält sie den kritischen Grundgedanken, sich nicht mit der vermeintlichen Funktionalität sozialer Verhältnisse (oder der Sozialontologie der wechselseitigen Subversion von Notwendigkeit und Kontingenz) zufrieden zu geben, sondern 
die Interpretation der Verhältnisse auf ihre Veränderbarkeit hin anzulegen und die Akteure insoweit als kompetente Akteure auch (im Doppelsinn) einzusetzen. Doch anders als Demirovic beharre ich in der Tat darauf, daß sich »kapitalistische Verhältnisse ... vom Konsens und den Alltagsgewohnheiten der Individuen « (Demirovic 1992, 148) abgelöst haben und im Rückgriff auf diese Gewohnheiten nicht zureichend verstanden werden können.

Die Orientierung an kompetenten Akteuren muß ergänzt werden durch die Berücksichtgung struktureller Zwänge, die in den sozialen Formen angelegt sind. Allerdings darf in der Tat dabei nicht der kritische Grundgehalt verloren gehen. Dies läßt sich abschließend an einem Beispiel demonstrieren: an den Veränderungen des Nationalstaats im Rahmen kapitalistischer Globalisierung. Ohne Zweifel ist der Verweis vieler Konzerne, z.B. der Automobilindustrie, auf die internationale Wettbewerbsfähigkeit und die Konkurrenzfähigkeit am »Standort Deutschland《 (auch) ein strategisches Argument, mit dem in sozialen Auseinandersetzungen Druck gemacht werden soll. Insofern wäre es in der Tat fatal, hier pauschal in vermeintlichem Realismus strukturelle ökonomische Zwänge in Anschlag zu bringen und dadurch die scheinbare Alternativlosigkeit des »Sachzwangs Weltmarkt « noch ideologisch zu überhöhen. Dessen Logik setzt sich in der Tat erst mit Hilfe eben der bewußten Strategien konkurrierender ökonomischer Akteure durch. Trotzdem sind sie angesichts der Krisensituation nur zum Teil als kompetente Akteure anzusehen. Vielmehr haben diese Strategien sehr viel mit den Veränderungen des Nationalstaats und der auf ihn bezogenen Form politischer Regulierung zu tun. Ohne seine Aushöhlung und die Transformation zum nationalen Wettbewerbsstaat (Hirsch 1995), d.h. ohne die Veränderung im strukturellen Terrain, auf dem sich diese Strategien bewegen, und das sie dadurch z.T. auch transformieren, lassen sich gesellschaftliche Entwicklungen nicht erklären. Und trotzdem unterscheiden sich kritische von realistischen Erklärungsvarianten, insofern diese die Entwicklungen noch affirmativ verdoppeln (Röttger 1995).

Mit dem Verweis auf soziale Formen und strukturelle Zwänge soll also der vermeintlichen Selbstgenügsamkeit ökonomischer Erklärungsstrategien genauso entgegengearbeitet werden wie der vermeintlichen Unabhängigkeit funktionaler Zusammenhänge von den handelnden Akteuren. Beide Aspekte verbindet der Begriff des »radikalen Reformismus«: Daß soziale Formen weder gänzlich unabhängig sind von den Handlungskapazitäten kompetenter Akteure wie diese historisch erst gegen die Dominanz struktureller Zwänge freigesetzt werden müssten. Das Verhältnis beider Dimensionen läßt sich so selbst als Widerspruch analysieren - und analysieren heißt hier: gerade nicht auf eine der beiden Seiten auflösen. Wenn für die- 
sen Zusammenhang in der Tradition dialektischer Gesellschaftstheorie der Begriff der Totalität verwendet wird, dann ist diese Einschränkung entscheidend." Totalität ist in diesem Sinne nach Adorno »eine Kategorie der Vermittlung« (Adorno 1979, 549). Die Funktionalität des gesellschaftlichen Zusammenhangs ist gerade nicht auf gesellschaftliche Strukturprinzipien reduzierbar, sondern ist als Widerspruch in kritischer Absicht, d.h. mit dem Ziel des Aufzeigens der Veränderbarkeit der Verhältnisse, auf den Begriff zu bringen. Schon Adorno war klar, daß in der kapitalistischen Gesellschaft nicht alles aus einem Prinzip zu deduzieren ist, sondern daß es ergänzend zur Verselbständigung ihrer Formen auch der relativen Selbständigkeit »ungezählte(r) nicht-kapitalistischer Enklaven« (ebd.) bedarf, deren Selbständigkeit heute aber von einer nicht-ständisch und nichthierarchisch stratifizierten Gesellschaftsform gerade auch ermöglicht wird. Gerade deswegen beharrt er auf dem Begriff der Totalität:

»Selbst jene Enklaven, die ungleichzeitigen Sozialgebilde, Favoriten einer Soziologie, die des Begriffs der Gesellschaft gleichwie eines allzu spektakulären Philosophems sich entledigen möchten, werden, was sie sind, nicht an sich, sondern erst in der Relation zu der herrschenden Totale, von der sie abweichen. Das diurte in der heute beliebtesten soziologischen Konzeption, der der middle range thcory, arg unterschätzt sein.« (ebd. 550)

Obwohl sich die Erwartungen nicht mehr zentral auf ungleichzeitige Phänome konzentrieren, sondern auf soziale Kämpfe und den relativen Eigensinn sozialer Prozesse, und der Anspruch einer »Theorie mittlerer Reichweite «, auf den sich die Regulationisten oft zurückziehen, zunächst einmal historisch gemeint ist, trifft dieses Zitat das Problem ziemlich genau: Der relative Eigenwert der verschiedenen Phasen des Kapitalismus, die Bedeutung institutioneller Konfigurationen für deren Ausgestaltung und die Rolle kompetenten Handelns in diesen Gestaltungsprozessen kann erst dann richtig verstanden werden, wenn die Vermittlungen freigelegt und der Totalitätsbezug entfaltet werden kann.

\section{Literatur}

Adorno, Theodor W. (1979): Soziologische Schriften 1, Frankfurt/M.

Backhaus, Hans-Georg (1996): Dic Irtümer der nationalökonomischen Marx-Kritik als Grundmängel der nationalökonomischen Theoriebildung, in: H.Brentel u.a. (Hg.): Gegensätze. Elemente kritischer Theorie, Frankfurt/M. (im Erscheinen).

Demirovic, Alex (1992): Regulation und Hegemonie, in: Demirovic u.a. (Hg.) (1992).

Demirovic, A.; Krebs, H.P,; Sablowski, T. (Hg.) (1992): Hcgemonie und Staat, Münster.

8 Un keine Mißverständnisse aufkommen zu lassen; Es soll kcineswegs behauptet werden, daß in der Adornoschen Gescllschaftstheorie für sich genommen schon die Lösung der hicr diskutierten Probleme enthalten ist. Daß hingegen die kritische Theorie gerade in ökonomietheoretischer Hinsicht und mit Blick auf soziale und politische Institutionen einige Defizite aufweist, ist schon öfter festgestellt worden (vgl. für die Ökonomictheorie jetzt: Johannes 1995). 
Elam, Mark (1994): Puzzling out the Post-Fordist Debate: Tecchnology, Markets and Institutions, in: A.Amin (Ed.) (1994): Post-Fordism, Oxford/Cambridge.

Esser, J,; Görg, Ch.; Hirsch, J. (1994): Politik, Institutionen und Staat. Zur Kritik der Regulationstheorie, Hamburg.

Esser, J,; Görg, Ch.; Hirsch, J.; Sablowski, T. (1994): Von den »Krisen der Regulation « zum »radikalen Reformismus«, in: Esser u.a. $(\mathrm{Hg})(1994)$.

Giddens, Anthony (1981): A Contemporary Critique of Historical Materialism. Vol 1, London.

Giddens, Anthony (1988): Die Konstitution der Gesellschaft, Frankfurt/M.

Görg, Christoph (1994a): Regulation - ein neues »Paradigmak? in: Esser u.a. (Hg) (1994).

Görg, Christoph (1994b): Der Institutionenbegriff in der »Theorie der Strukturierung«, in: Esser wa. $(\mathrm{Hg})(1994)$.

Görg, Christoph (1994c): Krise und Institution, in: Esser u.a. ( $\left.H_{g}\right)(1994)$.

Hirsch, Joachim (1994): Politische Form, politische Institutionen und Staat, in: Esser u.a. ( $\mathrm{Hg})$ (1994).

Hirsch, Joachim (1995): Der nationale Wettbewerbsstaat, Berlin.

Johannes, Rolf (1995): Das ausgesparte Zentrum. Adornos Verhältnis zur Ökonomie, in: G.Schweppenhäuser (Hg): Soziologie im Spätkapitalismus, Darmstadt.

Krebs, .P.; Sablowski, T. (1992): Ökonomie als soziale Regularisierung, in: Demirovic u.a. (Hg.) (I992).

Lipietz, Alain (1992): Vom Althusserismus zur »Theorie der Regulation«, in: Demirovic u.a. ( $\mathrm{Hg}$ ) (1992).

Mahnkopf, Birgit (1988): Soziale Grenzen fordistischer Regulation, in: dies. (Hg): Der gewendete Kapitalismus, Münster.

Müller, Hans-Peter (1992): Sozialstruktur und Lebensstile, Frankfurt/M.

Polanyi, Karl (1977): The Great Transformation, Wien.

Ritsert, Jürgen (1973): Probleme politisch-ökonomischer Theoriebildung, Frankfurt/M.

Ritscrt, Jürgen (1988): Das Bellen des toten Hundes, Frankfurt/M.

Röttger, Bernd (1995): Politökonomische Analysen globaler Beziehungen, in: links Nr. 304/305, S. 16-19.

Sablowski, Thomas (1992): Zum Status des Hegemoniebegriffs in der Regulationstheorie, in: Esser u.a. (Hg.) (1994).

Scherrer, Christoph (1995): Einc diskursanalytische Kritik der Regulationstheorie, in: PROKLA 100. 